\title{
SÄUGETIER-TERRITORIEN UND IHRE MARKIERUNG
}

\author{
vON
}

H. HEDIGER

Basel

Inbezug auf ihren subjektiven Raum haben Tier und Mensch viel miteinander gemeinsam (vgl. HolzAPFEL, I943), vor allem das Bestreben nach einer deutlichen Abgrenzung, ferner die Art der Differenzierung und die ausgesprochene Tendenz zum Verharren (vgl. Hediger, 1946). Selbst in der modernen Wohnung finden wir eine Aufteilung, wie sie auch bei vielen Tieren vorkommt; es gibt Schlaf-, Ess-, Bade-, Vorrats-, Kotstellen usw. Nur einen Raumteil, der selbst in der primitivsten Eingeborenenhütte niemals fehlt, vermissen wir beim Tier grundsätzlich: die Feuerstelle.

Hinsichtlich der Verbindungen zwischen den Örtlichkeiten mit speziell zugeordneten Funktionen hat von jeher nicht nur eine weitgehende Entsprechung bestanden, sondern zum Teil eine Überschneidung. Wohl erscheint uns heute die verkehrsreiche Strasse von einem Wildwechsel so verschieden, dass uns das Verwandte zwischen beiden Wegtypen meist gar nicht bewusst wird; aber wer etwa in Gegenden nachsieht, wo das Grosswild noch zahlreicher ist als der weisse Mensch, da lässt sich vielfach zwischen Menschenpfad und Tierwechsel überhaupt kein Unterschied feststellen - weil solche Wechsel heute noch von Eingeborenen und von Vertretern verschiedener Wildarten gemeinsam, manchmal gleichzeitig benützt werden.

Mit der Erforschung der tierlichen Strassensysteme stehen wir heute noch am Anfang. Es kann aber jetzt schon ausgesagt wenden, dass man auch beim Tier sozusagen den Typus der Überlandstrasse und den der Dorfgasse oder des privaten Gartenweges unterscheiden kann. Der erstgenannte ist im Tierreich dem grossen Raum-Zeit-System zugeordnet, das heisst den periodischen grossen Ortsveränderungen, die oft von bedeutenden Individuenmengen unternommen und trotz ihrer heterogenen Motivierung gewöhnlich einfach als „Wanderungen” bezeichnet werden (Aal, Lachs, Zugvogel, Ren, Antilopen usw.). Die kleinen, kurzen Wege dagegen sind dem kleinen Raum-Zeit-System zugeordnet, d.h. auf und längs diesen vollziehen sich die täglichen Gänge oder Flüge innerhalb des Territoriums und eventuell seiner näheren Umgebung.

Unter Territorium sei hier kurz der vom Individuum oder von einer orga- 
nisierten Vielheit von Individuen (Rudel, Herde, Schwarm, etc.) verteidigte Raumausschnitt verstanden. Das Territorium wird in typischer Weise kenntlich gemacht (markiert) und in erster Linie gegenüber arteigenen Rivalen, viel weniger oder gar nicht gegenüber artfremden Tieren verteidigt. Heute sind wir über Grösse und Markierungsmethode bei einzelnen Tierarten verhältnismässig gut unterrichtet; aber man sollte sich darüber klar sein, dass diese Tatsachen für jede Tierart ebenso bekannt sein müssten und zu ihrer Charakterisierung ebenso unerlässlich sind, wie etwa die Zahl ihrer Zehen, ihre Gebissformel oder andere morphologische Merkmale, die bisher ausschliesslich zur Beschreibung herangezogen zu werden pflegten.

Unzweifelhaft hat die ornithologische Territoriumsforschung heute einen bedeutenden Vorsprung aufzuweisen, daher soll es die Aufgabe dieses kleinen Aufsatzes sein, einige Säuger-Territorien etwas zu beleuchten.

Was zunächst die Territoriumsgrösse, d.h. die dem freilebenden Tier zur Verfügung stehende Raum-Quantität anbetrifft, so gibt z.B. LEOPOLD (I939, S. 233) eine interessante Aufstellung auf Grund der Verhältnisse im Santa Rita Range-Reservat in Arizona. Auf eine Quadratmeile kommen dort an Säugetieren u.a. I Coyote (Canis latrans), I5 Stinktiere, 25 weidende Rinder, 45 Kaninchen, I280 Känguruh-Ratten (Dipodomys), 6400 Wald-Ratten (Neotoma) und 17948 Mäuse verschiedener Artzugehörigkeit. In Kalifornien kommen etwa 360 Hirsche auf I Puma, usw. Aus diesen Daten geht eindrücklich hervor, I., dass die Territorien vieler Tierarten sich überschneiden, 2., dass kleine Tiere ein kleines, grosse Tiere ein grosses Territorium besitzen und 3., dass Raubtiere ein viel grösseres Territorium haben müssen als Pflanzenfresser. Sie müssen ja - als Selbstversorger - über so grosse Herden von Beutetieren verfügen, dass sie gut davon leben können, ohne sie zu dezimieren. Wenn ein Puma 360 Hirsche ,besitzt", so kann es jährlich mindestens $30 \%$, also gut Ioo Stück „konsumieren”, d.h. jeden 3. oder 4. Tag eines. Natürlich lebt ein Puma nicht ausschliesslich von Hirschen und der Hirschbestand ist nicht ausschliesslich ihm reserviert. Allzu genau dürfen also solche Zahlen nicht genommen werden, aber sie geben doch einen instruktiven Begriff von der Grössenordnung.

Interessant sind die Schätzungen von Hubert (I947, S. 3 I ff) über die Wohndichte verschiedener Grosswildarten im Albert-Nationalpark im Belgischen Kongo. Dort soll I Löwe auf $3-4 \mathrm{~km}^{2}$ kommen, 3 Flusspferde auf I $\mathrm{km}^{2}, 3$ Büffel auf $2 \mathrm{~km}^{2}$, I Warzenschwein auf $6 \mathrm{~km}^{2}$ und I Elefant auf $8 \mathrm{~km}^{2}$. I93I soll das Wild sich folgendermassen verteilt haben:

12 Topi-Antilopen

24 Cob-Antilopen

2 Riedböcke pro $\mathrm{km}^{2}$.

Nach einer anderen Kalkulation traf es:

I Wasserbock auf I $\mathrm{km}^{2}$

I Buschbock auf $2 \mathrm{~km}^{2}$ 
I Warzenschwein auf $\mathrm{I} \mathrm{km}^{2}$

I Löwe auf $5 \mathrm{~km}^{2}$

I Fleckenhyäne auf $4 \mathrm{~km}^{2}$

I Leopand auf $12 \mathrm{~km}^{2}$

Auch hier erwiesen sich die Raubtier-Territorien naturgemäss als wesentlich grösser als die der Pflanzenfresser.

Was nun die Inneneinrichtung des Territoriums, also die Raum-Qualität

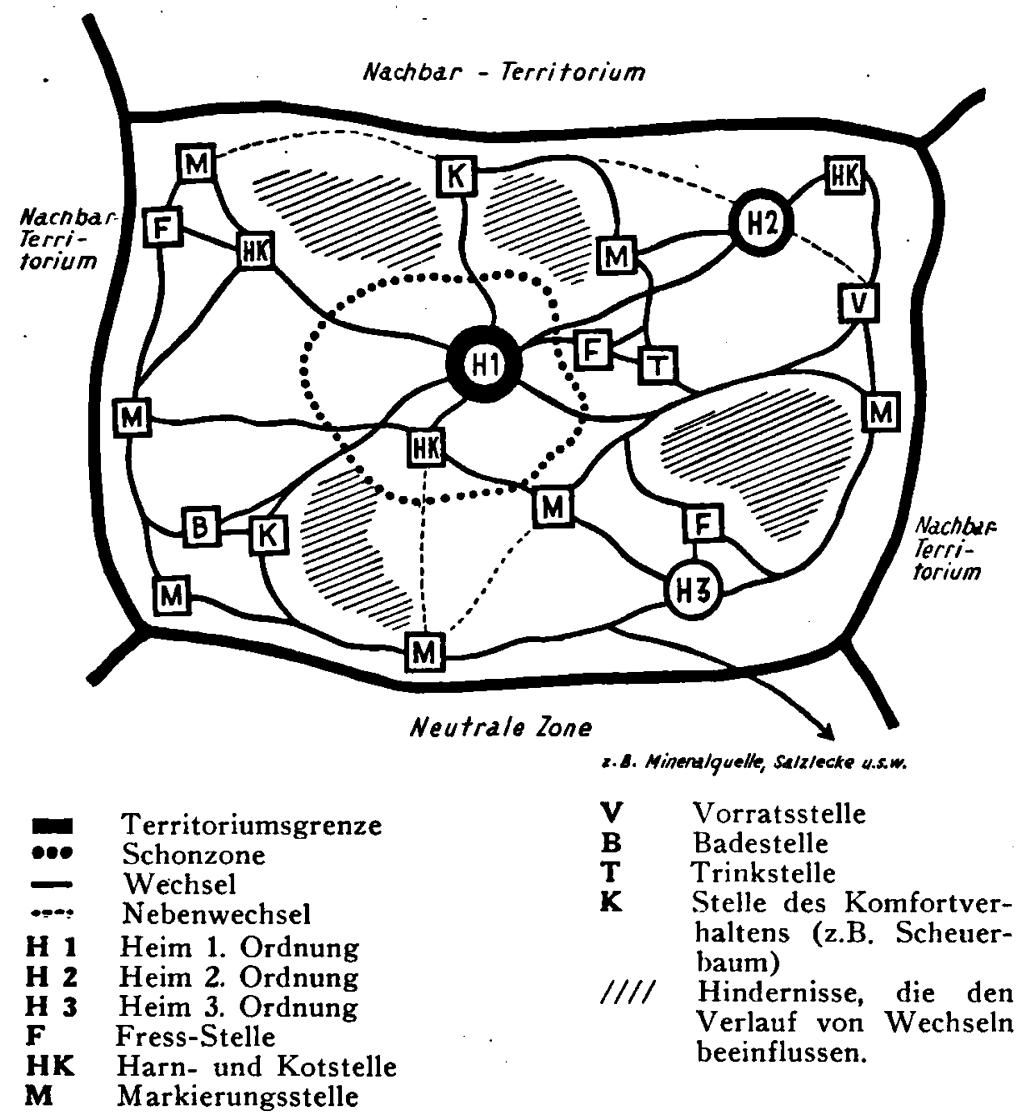

Abb. 1. Schema eines Territoriums

anbetrifft, so habe ich bereits 1942 versucht, das Schema eines Säuger-Territoriums mit seiner Inneneinrichtung zu skizzieren. Heute lassen sich dieser Darstellung (Abb. I), deren Schema-Charakter neuerdings unterstrichen sei, vielleicht noch zwei oft $z u$ beobachtende Merkmale einfügen, nämlich die Schon-Zone und die neutrale Zone. Die Schon-Zone liegt immer im Territorium drin und bildet einen Raumausschnitt, der - wenn er überhaupt auftritt - meistens das Heim, also das Lager, das Nest, den Bau usw. umgibt. Das Heim wurde als der Ort maximaler Geborgenheit definiert. In dieser 
Schon-Zone, die, wie gesagt, oft aber durchaus nicht immer auftritt, verzichtet das Tier auf Nahrungserwerb, gleichgültig ob es sich um Pflanzenfresser oder Raubtiere handelt.

Wie ich mich selber oft überzeugen konnte, schont z.B. der Hausmarder (Martes foina) sehr oft die Taubenschläge und Hühnerställe, die sich unmittelbar neben seinem Heim befinden. Für viele andere Raubtiere und auch für Raubvögel ist Entsprechendes festgestellt; aber auch manche Pflanzenfresser beginnen erst in einer bestimmten Entfernung von ihrem Heim mit Äsen von Gras, Laub etc. Die unmittelbare Umgebung ihres Heimes ist in der Regel nie kahlgefressen. Das Erdferkel (Orycteropus) schlägt die Termitenstöcke direkt neben seinem Erdbau nicht an, sondern es beginnt mit der Nahrungssuche erst bei entfernteren Termitenstöcken.

Eine Erklärung für das Vorkommen von Schon-Zonen ist nicht leicht zu geben. Bei Raubtieren könnte man sich vorstellen, dass in der näheren Umgebung des Heimes richtige Jagdstimmung gar nicht aufzukommen vermag, dass also „das Einklinken in den Funktionskreis der Beute" - um mit UexkülL zu sprechen - im Felde der Heimwirkung nicht möglich ist. Bei Pflanzenfressern ist zu bedenken, dass ohne Schonung der Vegetation das Heim durch das Kahlfressen seiner Umgebung sehr stark exponiert würde. Vielen Tieren eignet ein starkes Bedürfnis nach Deckung; sie werden diese nicht gerade dort vernichten, wo sie am notwendigsten ist.

Vielleicht spielt bei Pflanzenfressern auch der Umstand eine Rolle, dass die Gräser, Blätter usw. in der Umgebung des Heimes, also an der meistbegangenen Stelle des Territoriums, so stark mit körpereigenen Geruchsstof fen imprägniert sind, dass sie ihre Futter-Valenzen eingebüsst haben. In Tiergärten kann man bei Huftieren (Zwergziegen, Antilopen u.a.) gelegentlich die Beobachtung machen, dass ihre Ausläufe einen bescheidenen Rasen aufweisen, der von ihnen nicht beachtet, bzw. nicht abgeweidet wird. Gibt man jedoch solchen Tieren Gras von gleicher Beschaffenheit, das aber nicht aus ihrem Gehege stammt, dann wird es meistens ohne weiteres gefressen. Die aus diesem Tatbestand sich ergebenden Fragen sind dem Experiment zugänglich; mit ihrer Bearbeitung ist im Basler Zoo bereits begonnen worden.

Hier sollen zunächst einige Beobachtungen über die Markierungsmethoden angeführt werden. Grundsätzlich lassen sich wohl die vier folgenden unterscheiden (vgl. I948, S. I I) :

I. Optische

2. Akustische

3. Olfaktorische

4. Kombinierte Methoden.

Im Tierreich sind diese Methoden weit und unregelmässig verteilt; so kommt z.B. die optische schon bei gewissen Wirbellosen vor (Winkerkrabbe Uca), aber auch bei manchen Fischen, Reptilien, vielen Vögeln (Erithacus) und einigen Säugetieren (z.B. Giraffe). Diese optische Methode lässt sich 
leicht gliedern in ein mehr statisches und ein dynamisches Verfahren. Ausgesprochen dynamisch ist etwa das Verhalten der Winkerkrabbe, welche durch rhythmisches Heben und Senken der grellgefärbten Signalschere ein überaus auffälliges bewegtes Zeichen übermittelt. Von statisch-optischer Markierung hingegen wäre etwa bei der Giraffe zu sprechen. Merkwürdigerweise ist früher die Färbung dieses riesigen Savannengeschöpfes als eine tarnende dargestellt worden; aber es hat in Wirklichkeit nie an Feldbeobachtern gefehlt, welche im Gegenteil die optische Auffälligkeit der Giraffe im Gelände geschildert haben; so führt z.B. Th. Roosevelt (19ro, S. 439) aus: „Sie ist eins der auffallendsten Tiẹe in der Natur. Eingeborene Jäger der echten Jägerstämme entdeckten sie stets in erstaunlicher Ferne, und in der Nähe entgeht sie ihren Augen nie; waś das Verbergen anbetrifft, so bringt ihre Färbung ihr nicht den geringsten Nutzen."

Nach meinen eigenen Beobachtungen in Giraffen-Biotopen bin ich überzeugt, dass diese Tiere über Kilometer hinweg miteinander in Sichtfühlung stehen und dass ihr auffälliges Muster dazu dient, die Beziehungen der Individuen eines Rudels und auch die verschiedenen Rudel untereinander zu regeln, ähnlich wie z.B. Brüllaffen (Alouatta) im südamerikanischen Urwald durch ihre Stimme im Rudel sowohl wie zwischen den Rudeln akustisch Fühlung nehmen. Unterstützt wird diese Annahme für die Giraffe noch dadurch, dass sie sich durch eine ungewöhnliche Armut an Hautdrüsen auszeichnet.

Bei den meisten Säugetieren erfolgt die Territoriums-Markierung auf olfaktorischem Wege, d.h. mit Hilfe von Duftstof fen, die entweder von besonderen Drüsen oder durch Kot und Harn geliefert werden. Da die meisten Säugetiere Makrosmaten sind, kann die Bevorzugung der geruchlichen Methode nicht wunder nehmen. Umso auf fälliger ist hingegen das Fehlen einer Duftstoffproduktion bei der - ihrer ganzen Organisation nach — stark optisch orientierten Giraffe. Auch bei einem anderen optisch organisierten Steppensäuger mit äusserst auffälliger Färbung - dem Zebra - muss ich annehmen, dass die Territoriums-Markierung auf dieselbe optisch-statische Art erfolgt wie bei der Giraffe. Ihr Muster wirkt an sich, wo sie auch gehen und stehen; es braucht hier - im Gegensatz etwa zur Winkerkrabbe - keine dynamische Signalbewegung:

In diesem Zusammenhang wirkt es sehr bezeichnend, dass die Giraffe allgemein als stumm gilt; sie ist es zwar nicht absolut. Ferner fällt auf, dass sich die imposantesten Lautäusserungen bei Säugern nicht etwa bei Makrosmaten finden, sondern im Gegenteil bei den verhältnismässig wenigen Mikrosmaten, z.B. bei den Affen. So ausgefallene Resonanzapparate wie z.B. beim Brüllaffen mit seinem blasig aufgetriebenen Zungenbein, oder beim Nasenaffen mit seiner grotesken Nase oder beim Orang Utan mit seinem riesigen Luftreservoir (Kehlsack) gibt es bei Makrosmaten nicht. Einge von ihnen verfügen allendings auch über bedeutende akustische Mittel, z.B. der Brunfthirsch, der Löwe usw. Ạber gerade das Löwengebrüll ist ja in seiner psycho- 
logischen Bedeutung noch recht schwer deutbar. - Die Methoden der Territoriums-Markierung vikarieren weitgehend.

Da der olfaktorischen Territoriums-Markierung bei Säugern eine so grosse Bedeutung zukommt, seien hier einige Einzelheiten erwähnt. In den letzten Jahrzehnten ist - namentlich durch Pocock (I9IO) und neuerdings durch SCHUMACHER (I943) - eine überraschende Fülle von Hautdrüsen bekannt geworden; viele waren vorher schon bekannt und immer 'wieder werden neue hinzuentdeckt. Heute wissen wir, dass es am Säugetierkörper sozusagen keine Stelle gibt - vom Kopf bis zum Schwanz, vom Fuss bis zum Rücken - an der nicht bei 'irgendeiner Art eine Duftdrüse auftreten könnte, die sich mit der Territoriums-Markierung in Zusammenhang bringen lässt. Natürlich darf erst dann von Drüsen im Dienst der Territoriums-Markierung gesprochen werden, wenn das für jede einzelne Art durch direkte Beobachtung oder noch besser - auf Grund von Experimenten bestätigt worden ist. Indessen braucht die Zurückhaltung mit der Interpretation auch nicht übertrieben zu werden, da auf Grund eines viele untersuchte Einzelfälle umfassenden Überblicks doch gewisse Analogieschlüsse ohne allzugrosses Risiko gezogen werden dürfen. Wenn beispielsweise heute ein neues Huftier entdeckt würde, das im männlichen Geschlecht in der Kopfregion eine stark sezernierende Hautdrüse (Duftdrüse) aufweist, so darf mit einer gewissen Berechtigung angenommen werden, dass ihr Sekret wie in Dutzenden von anderen Fällen, der olfaktorischen Markierung dient.

Hier seien aus der Fülle nur einige Beispiele angeführt, die ich z.T. im Freileben, z.T. im Zoo etwas näher beobachten konnte. Jeder, der Säugetiere in mehreren Arten um sich hat, wird in der Lage sein, die angedeutete Liste fortzusetzen. $\mathrm{Zu}$ den Parade-Beispielen, die ich den Studenten vorzuführen pflegte, gehört die indische Hirschziegenantilope (Antilope cerzicapra, Abb. 2) und der ostafrikanische, kurzschwänzige Mungo (? Mungos spec., Abb. 3). Die männlichen Hirschziegenantilopen haben so grosse Antorbitaldrüsen, dass man leicht die Spitze des Zeigefingers in die geöffnete Mündung einführen könnte. Das Tier hat die Möglichkeit, die Drüsenöffnung willkürlich weit zu öffnen oder ziemlich weitgehend zu schliessen. Die starke Bindung der Hirschziegenantilope an ihr Territorium ist in Indien so bekannt, dass sie praktisch ausgewertet wird durch die Anwendung einer besonderen Jagdmethode, in der die Territoriums-Verteidigung schliesslich dem Bock zum Verhängnis wird (vgl. I948, S. 50). Bei dieser Tierart ist keine bestimmte Brunft- und Setzzeit ausgebildet; die Weibchen können zu jeder Jahreszeit in Brunft, die Jungen demnach während des ganzen Jahres zur Welt kommen. Daher is ${ }^{2}$ die Territorium-Verteidigung für den Bock dauernd aktuell; bei ihm ist deshalb keine rhythmische Funktionssteigerung oder -abnahme der „Territorium-Drüse” zu beobachten. Er muss die Duftmarken seines und seines Rudels Wohnraumes immer wieder auffrischen dadurch, dass er an vorstehenden $Z$ weigen, steifen Halmen usw. seine weitgeöffnete Antorbitaldrüse so hin und her reibt, dass Bijdragen tot de Dierkunde, Afl, 28. 
beträchtliche Sekretportionen daran haften bleiben (Abb. 4). Die strömen sogar für die menschliche Nase einen intensiven charakteristischen Duft aus. In Basler Zoo fand ich ganze Klumpen des schwarzen, wachsartigen Sekretes an einzelnen Eisenstangen des Geheges, besonders auch an dem vom Wärter immer wieder berührten Schloss. Als ich einige geeignete Äste im Gehege befestigte, wurden diese sofort entsprechend verwendet: mit vorsichtigen

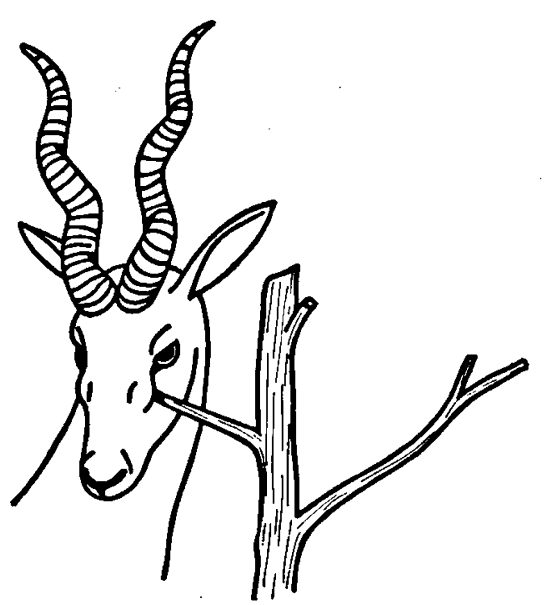

Abb. 2. Schematische Zeichnung eines markierenden Bockes der Hirschziegenantilope (Antilope cervicapra): Die geoffnete Antorbitaldrüse wird über einen Zweigstumpf gestülpt und auf diese Weise Sekret daran abgegeben

Abb. 3. Ostafrikanischer Mungo (?Mungos spec.) in typischer Markierungsstellung : durch Handstand wird die Analdrüse mit der Ast-Unterseite in Kontakt gebracht (Nach einer Photographie gezeichnet)

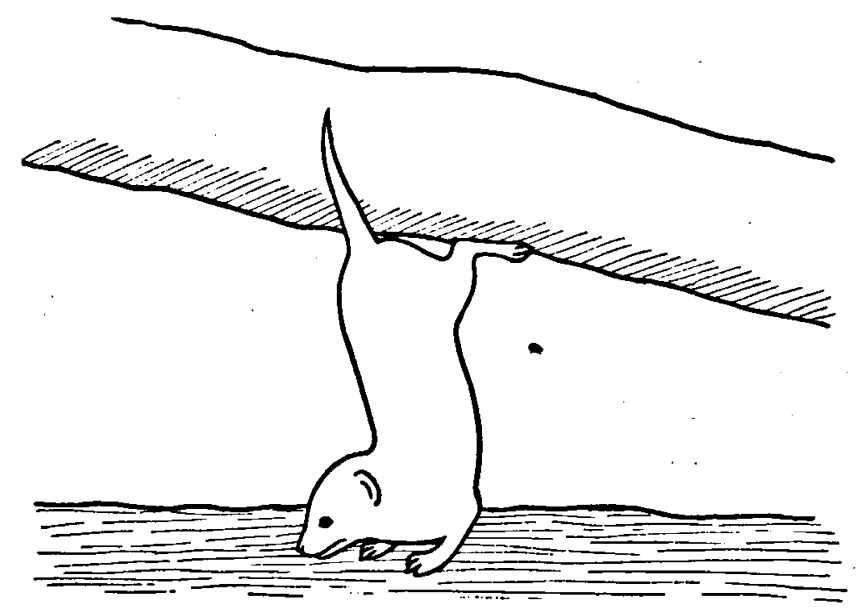

Kopfbewegungen stülpte der Antilopenbock seine stark geöffnete Drüse über den Aststumpf, der zum Anbringen des Sekretes gewählt worden war.

Beim Gemsbock (Rupicapra rupicapra) liegen grundsätzlich ähnliche, in Einzelheiten jedoch abweichende Verhältnisse vor: Erstens wird die Territoriums-Markierung für ihn besonders während der Brunftzeit, im November/ Dezember, bedeutsam. Während dieser Zeit nur sind seine Markierungsdrüsen funktionell. Zweitens liegen diese nicht antorbital, sondern retrocornual, d.h. 
unmittelbar'hinter dem Gehörn. Auf die Brunftzeit hin schwellen diese Drüsen stark an, in der Form etwa wie Walnüsse, und beginnen zu sezernieren. Das Sekret wird weniger an vorstehenden Aststümpfen und nicht in so bedeuten len Mengen abgestrichen wie bei der Hirschziegenantilope, sondern in geringfügigen Quantitäten an ganz feinen Zweigen, auch an verhältnismässig kurzstieligen Kräutern usw. (Abb. 5, 6). Früher wurden die Brunftfeigen

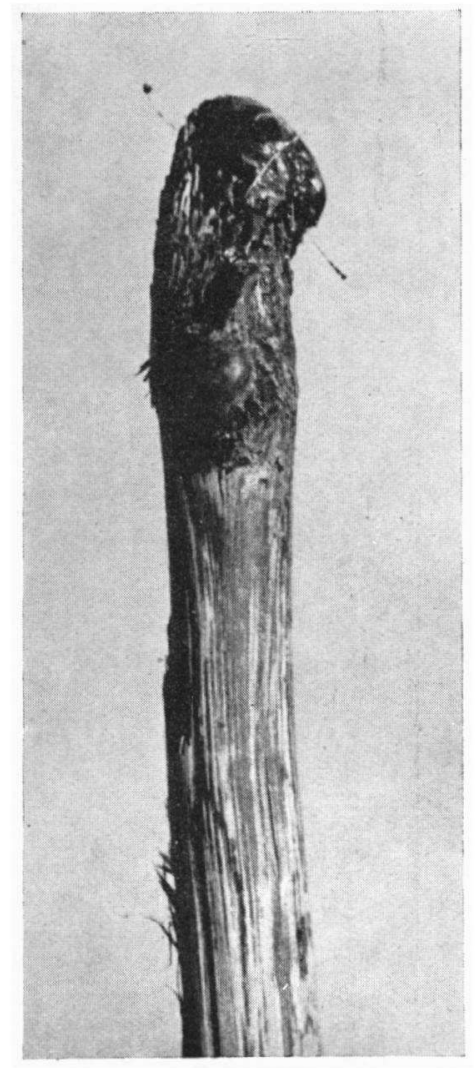

Abb. 4. Aststumpf mit Sekretmasse aus dem Antorbitalorgan eines Hirschziegenantilopen-Bockes (Photo Hediger)

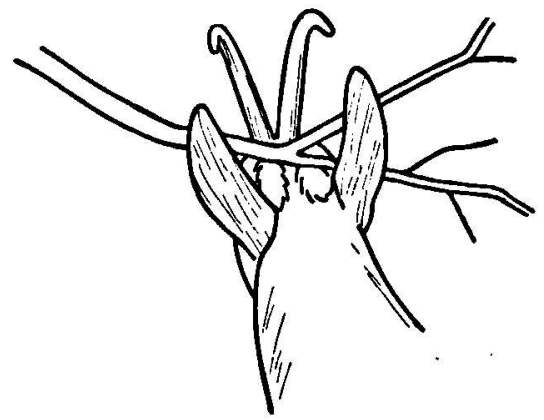

Abb. 5. Gemsbock seine Brunftfeigen (Retrocornualdrüsen) an einem Zweig streifend (Nach einer Photo gezeichnet)

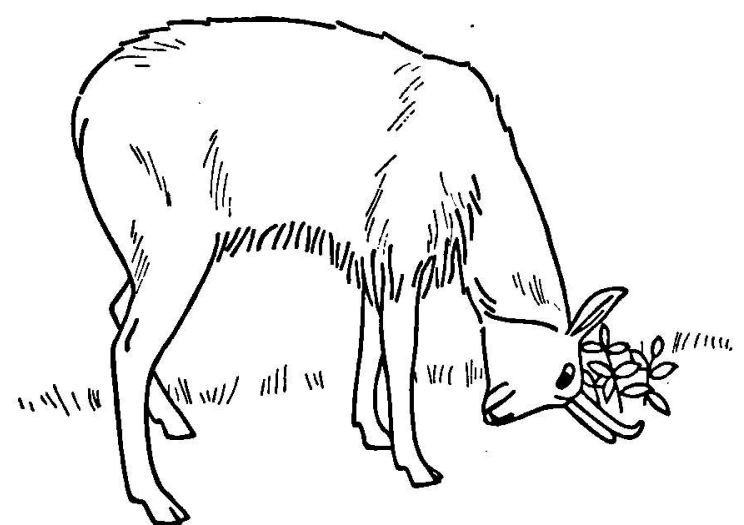

Abb. 6. Gemsbock Sekret aus seinem Retrocornualdrüsen an niederem Kraut abstreifend. Wie in Abb. 5 handelt es sich um einen in Gefangenschaft beobachteten Gemsbock, dem die Gehörnspitzen abgesägt worden sind (Nach einer Photo gezeichnet)

von den Jägern bezeichnenderweise als accessorische Atemorgane gedeutet, welche den Hochgebirgstieren das Atmen in der dünnen Luft erleichterten. Bei den weiblichen Gemsen sind diese Retrocornualdrüsen übrigens auch deutlich ausgebildet, jedoch viel kleiner als bei den Böcken und zu einer Sekretion kommt es nicht.

Überaus reich an Hautdrüsen sind die Marderartigen (Musteliden). Rüden des Baum- und Hausmarders pflegen mit dem Hinterteil in charakteristischer 
Weise über Unebenheiten oder vorstehende Teile von Ästen herumzurutschen und dabei von ihrem Analdrüsensekret abzustreifen, wie F. Goethe (1938) ausgeführt hat. Solche Duftmarken finden sich immer auf den Ast-Oberseiten (Abb. 7). Das Gegenstück dazu bildet in gewissem Sinne der ostafrikanische Mungo, eine kleine, kurzschwänzige, nicht genau bestimmte Art aus Nairobi, die ich längere Zeit im Basler Zoo beobachten konnte. Diese Art bringt ihre Duftmarke nämlich nicht auf der Ober-, sondern stets an der Unterseite von Ästen an, und zwar beteiligen sich hier Männchen und Weibchen unter Umständen gleichzeitig an- der Markierung. Das ist der einzige derartige Fall, von dem ich Kenntnis habe; sonst ist die Territorium-Markierung ausschliesslich eine Angelegenheit des männlichen Geschlechts. Sie kann ja dabei geradezu einen Sexualdimorphismus bedingen, wie wir z.B. beim Zwergflusspferd (Choeropsis) noch sehen werden. Um das Verhalten des Mungos zu demonstrieren, brauchte man nur die niedrigen Kletteräste auf ihrer Unterseite mit einem feuchten Lappen abzuwischen. Dann kamen sofort die beiden zahmen Mungos schnuppernd herbei, richteten sich auf und beschnupperten die abgewaschene Astunterseite ausgiebig. Darauf machten sie - zuweilen beide gleichzeitig - einen regelrechten Handstand und pressten bei dorsal umgelegtem Schwanz die vorgestülpte Analregion an die Astunterseite, um Sekretportionen aus ihren Analdrüsen daran anzuheften (Abb. 3). Bei langschwänzigen Mungo-Arten konnte ich dieses Verhalten nie beobachten.

Wie erwähnt, ist die Territorium-Markierung — sei sie optisch, akustisch oder olfaktorisch — in der Regel Sache des Männchens. Wenn besondere Organe für diese Funktion ausgebildet sind, kann das zu einem Sexual-Dimorphismus führen. Das ist der Fall z.B. beim westafrikanischen Zwergflusspferd (Choeropsis liberiensis), wahrscheinlich auch bei Hippopotamus, was ich jedoch nicht genau nachprüfen konnte.

Beim Zwergflusspferd werden Kot und Harn als Duftstoffe für die Territorium-Markierung benützt und zwar werden sie in feinen Partikeln möglichst weit verteilt, sodass schliesslich der ganze Wohnraum dieser Tiere mit ihrem individuellen Duftstoff mehr oder weniger imprägniert ist. Im Zoo fliegen Te:lchen davon mehrere Meter weit nach allen Richtungen, auch aufwärts bis an die Decke des Stalles. Der Markierungsakt besteht darin, dass der ausserordentlich muskulöse, zwiebelförmige und mit einem pinselartigen Borstenbesatz versehene Schwanz des Bullen (Abb. 8) in eine schwirrende Bewegung versetzt und der aus dem Anus austretende Kot nach allen Richtungen verspritzt wird. Gleichzeitig tritt aus dem düsenförmigen Penis ein kräftiger Harnstrahl, der nach hinten oben direkt auf den vibrierenden Schwanz gerichtet ist, was ein wirksames Verspritzen des Harnes und seine Vermischung mit Kotpartikeln zur Folge hat. Das Weibchen hat einen. viel weniger kräftigen, nur mit wenigen kurzen Haaren versehenen Schwanz (Abb. 9) ; es setzt Kot und Harn unverteilt am Boden ab.

Beim grossen Flusspferd (Hippopotamus) konnte ich dasselbe Verhalten 
auch im Freien beobachten. Überlegene Bullen können schwächeren Eindringlingen Kot und Harn geradezu ins Gesicht sprengen, worauf sich die abgewiesenen Rivalen zurückziehen.

Das Harnversprühen nach rückwärts kommt auch bei vielen andern Tieren vor, so z.B. bei Nashorn, Tapir, Tiger, Löwe, Gepard, Sumpfbiber, Wild-

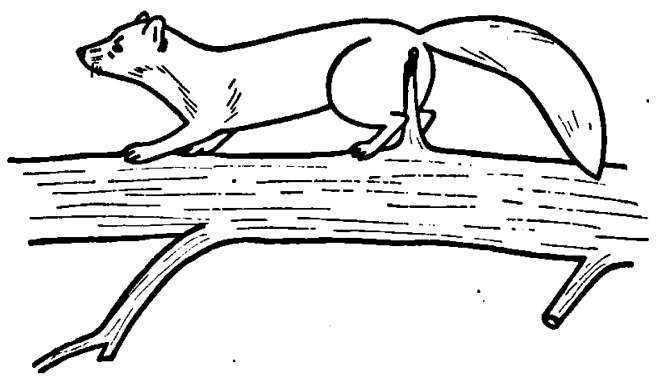

Abb. 7. Hausmarder (Martes foina) in typischer Markierstellung: Abstreifen des Analdrüsensekretes an einem $Z$ weigstumpf auf der Astoberseite.

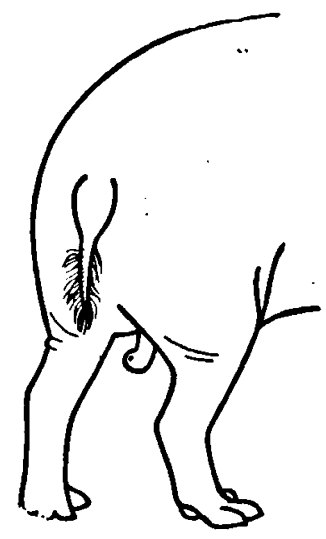

Abb. 8. Hinterteil eines männlichen Zwergflusspferdes (Choeropsis liberiensis) mit dem muskulösen und stark beborsteten Schwanz und dem düsenartig nach hinten oben (auf den Schwanz) gerichteten Penis (Nach einer Phote gezeichnet)

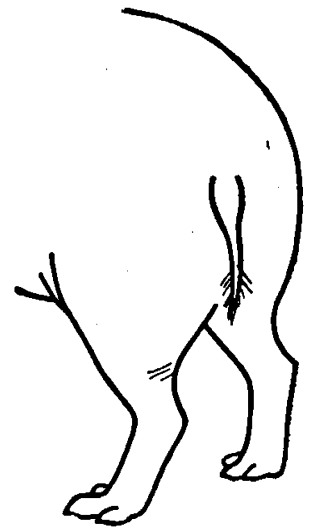

Abb. 9. Hinterteil eines weiblichen Zwergflusspferdes mit weniger muskulösem und weniger stark beborstetem Schwanz (Nach einer Photo gezeichnet)

kaninchen usw. Aus Analogie dürfen wir hier zweifellos annehmen, dass diese sonderbaren Konstruktionen und Verhaltensweisen der Territorium-Markierung dienen.

Manche Tiere, namentlich Huftiere, haben ihre besonderen Malbäume, an denen sie sich immer wieder scheuern, so z.B. Wildschweine, Wisent, Bär etc. Vermutlich kommt solchen Stellen nicht so sehr optische, als geruchliche Bedeutung zu; denn durch das hartnäckige Schaben an Bäumen entstehen nicht nur gut sichtbare, oft spezifisch umschriebene Rindenschäden, sondern es 
werden vielfach auch Geruchsstoffe bei dieser Gelegenheit deponiert. So konnten wir z.B. beim Braunbären feststellen, dass er während se:nem charakteristischen Rückenreiben an bestimmten Baumstämmen auch Harn abgibt (Abb. IO). Oft wälzt er sich mit der Schultergegend darin, um nachher mit dieser imprägnierten Partie wiederum am Stamm hin und her zu reiben. Auf Grund bestimmter Verhaltensweisen hatte ich früher den Verdacht, dass sich beim Braunbären auf dem Rücken zwischen den Schulterblättern eine Geruchsdrüse befinden müsse, indessen gelang es mir trotz der Untersuchung

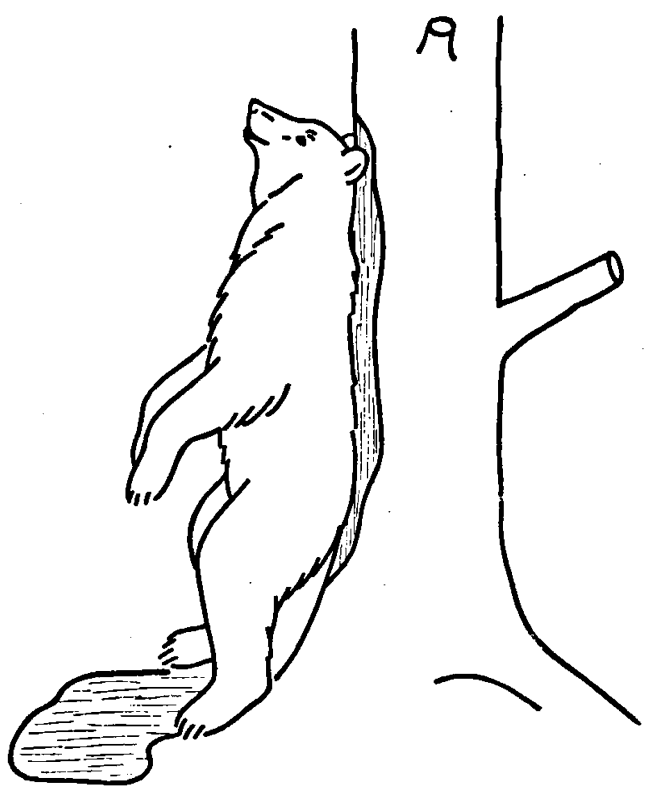

Abb. IO. Braunbär an seinem Schabebaum den Rücken reibend und gleichzeitig Harn abgebend (Nach einer Photo gezeichnet)

frischer Kadaver und abgezogener Felle nie, die vermutete Drüse nachzuweisen. Heute glaube ich, dass es sich hier - wie in vielen anderen Fällen nicht um eine Stelle handelt, wo Duftstoffe primär produziert werden, sondern um eine solche, wo anderwärts produziertes Material sekundär (durch das Wälzen im Harn) hingelangt, um von dort tertiär an die Markierungsstelle am Baum abgegeben zu werden. - Beim afrikanischen Mähnenschaf ( $\mathrm{Am}$ motragus lervia) konnte ich beobachten, dass sich die Böcke mit ihren spitzschaufelartigen Gehörnenden harnimprägnierte Erde auf den Rücken befördern.

Ähnlich wie der Bär verhält sich der Wisent (Bison bonasus) an seinem Malbaum. Dieser wird zunächst auf zweierlei Weise bearbeitet: Erstens schabt der Wisentstier mit der Vorderseite seines Horns am Baum, sodass ein charak. teristischer Rindendefekt entsteht, und zweitens mit der Rückseite seiner Hör- 
ner (Abb. I I). Gewöhnlich lässt der Bulle ausgiebig Harn in eine muldenartige Vertiefung in der Nähe plätschern und wälzt sich dann so darin, dass er zuweilen alle Viere in die Luft streckt. Mit dem so imprägnierten Buckel wird nun wieder an der Baumnarbe hin und her gerieben, sodass zweifellos bedeutende Mengen von Geruchsstof fen dorthin gebracht werden (Abb. I2). Beim Zusammenlassen von Wisenten im Zoo, wo sie während einiger Zeit getrennt

Abb. II. Wisent-Bulle mit der hinteren Hornseite an seinem Malbaum scheuernd (Nach einer. Photo gezeichnet)

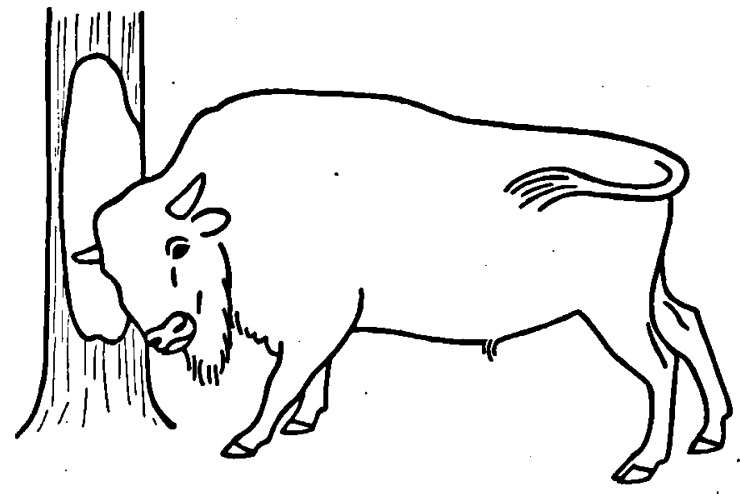

Abb. 12. Wisent-Bulle nachdem er sich in Rückenlage ausgiebig im eigenen Harn gewälzt hat, seinen Buckel am Malbaum fegend (Nach einer Photo gezeichnet)



untergebracht waren, fiel uns auf, dass sich die Tiere zunächst ,übersahen”, hingegen sich zu allererst dem ausgiebigen Beschnuppern der gegenseitigen Malbäume widmeten.

Sicher ist, diss Kot und Harn vom Tier nicht lediglich aus "Notdurft" abgegeben werden, sondern dass das oft sehr wichtige Bedeutungsträger sind, die für die Inneneinrichtung; besonders für die Kennzeichnung des Territoriums wichtig sind, wie ich bereits $1944 \mathrm{zu}$ zeigen versuchte.

Nachdem einmal die Bedeutung von Duftsekreten bei vielen Säugetieren als olfaktorisches Mittel der Territorium-Markierung erkannt war, wurden viele bisher unverständliche Verhaltensweisen mit einem Schlage klar, und es darf wohl behauptet werden, dass die Entdeckung des Markierungsverhaltens uns dem Verständnis tierlichen Verhaltens näher gebracht hat als manches Jahr 
vorausgegangener Forschung. Es wäre nur natürlich, wenn unter dem Einfluss der Auffindung dieser ungeheuer wichtigen Verhaltensregel in einzelnen Fällen etwas über das Ziel hinausgeschossen würde, wenn z.B. Sekretabgaben aus Analogie als Markierungsakt interpretiert würden, während sie in Wirklichkeit eine andere oder auch mehrere weitere Bedeutungen haben. Hier werden einze'ne Korrekturen unvermeidlich sein; manche Duftzeichen werden z.B. auch der eigenen Orientierung im Raum dienen. Wichtig war indessen, dass von verschiedenen Autoren überhaupt einmal Duftstoffe und Duft drüsen als für das Tiersubjekt in dieser oder jener Richtung äusserst bedeutsam aufgezeigt worden sind.

\section{LITERATUR}

GoEthe, F., 1938. Beobachtungen über das Absetzen von Witterungsmarken beim Baummarder. Der Deutsche Jäger, Nr. I3.

Hedrger, H., 1942. Wildtiere in Gefangenschaft. Ein Grundriss der Tiergartenbiologie. Basel.

- - I944. Die Bedeutung von Miktion und Defäkation bei Wildtieren. Schweiz. Zs. f. Psychol., Bd. 3, S. 170-182.

-, I946. Bemerkungen zum Raum-Zeit-System der Tiere. Desgl., Bd. 5, S. 24I-269.

- I948. Kleine Tropen-Zoologie. Acta Tropica, Supplementum-1. Basel.

Holzapfez-MeYer, M., 1943. Affektive Grundlagen tierischen Verhaltens. Schweiz. Zs. f. Psychol. Bd. 2.

Hubert, E., 1947. La faune des grands mammifères de la plaine Rwindi-Rutshuru. I.P.N.C.B. Bruxelles.

Leopold, A., 1939. Game Management. New York.

Pocock, R. I., 1910. On the specialised cutaneous glands of ruminants. Proc. Zool. Soc. Londen, S. 840-886.

Roosevelt, Th., I9IO. Afrikanische Wanderungen. Berlin.

Schumacher, S. v., 1943. Die Duftorgane (Hautdrüsenorgane) unseres Haarwildes. Zs. f. Jagdkde, Bd. 5 . 